\title{
Study on CA-CFAR Algorithm Based on Normalization Processing of Background Noise for HI of Optical Fiber
}

\author{
Yanping WANG, Dandan QU*, Chao ZHAO, and Dan YANG \\ School of Electrical and Information Engineering, North China University of Technology, Beijing 100144, China \\ *Corresponding author: Dandan QU \\ E-mail: qdd1962@163.com
}

\begin{abstract}
Optical fiber pre-warning system (OFPS) is often used to monitor the occurrence of disasters such as the leakage of oil and natural gas pipeline. It analyzes the collected vibration signals to judge whether there is any harmful intrusion (HI) events. At present, the research in this field is mainly focused on the constant false alarm rate (CFAR) methods and derivative algorithms to detect intrusion signals. However, the performance of CFAR is often limited to the actual collected signals distribution. It is found that the background noise usually obeys non-independent and identically distribution (Non-IID) through the statistical analysis of acquisition signals. In view of the actual signal distribution characteristics, this paper presents a CFAR detection method based on the normalization processing for background noise. A high-pass filter is designed for the actual Non-IID background noise data to obtain the characterization characteristic. Then, the background noise is converted to independent and identically distribution (IID) by using the data characteristic. Next, the collected data after normalization is processed with efficient cell average constant false alarm rate (CA-CFAR) method for detection. Finally, the results of experiments both show that the intrusion signals can be effectively detected, and the effectiveness of the algorithm is verified.
\end{abstract}

Keywords: OFPS; HI; CA-CFAR; normalization; Non-IID

Citation: Yanping WANG, Dandan QU, Chao ZHAO, and Dan YANG, "Study on CA-CFAR Algorithm Based on Normalization Processing of Background Noise for HI of Optical Fiber," Photonic Sensors, 2018, 8(4): 341-350.

\section{Introduction}

Oil and natural gas leakage caused by pipeline damage can lead to serious environmental pollution and economic loss [1-3]. Hence, it is urgent to find an effective pre-warning mode with rapid detection and precise location. Due to its advantage of long-distance detection, high sensitivity, anti-preventing electromagnetic interference, and timely warning to the pipeline damage, the optical fiber pre-warning system (OFPS) has attracted the attention of many scholars [4-7]. The specific processing is as follows. Firstly, the optical fiber is laid just above the oil pipeline in parallel. Secondly, the laser generated by the system hardware module is transmitted in the optical fiber medium, and then the collected backscattered light is analyzed to judge the occurrence of harmful intrusion. Finally, the related processed results are transmitted to a host computer for real-time displaying. This paper adopts the algorithm to accomplish analysis and detection for optical fiber signals.

It is known that the constant false alarm rate (CFAR) is a common target automatic detection method for radar signal processing [8]. Among them, the cell average constant false alarm rate (CA-CFAR) method is widely used in optical fiber vibration signals processing with the advantages of strong

Received: 11 March 2018 / Revised: 29 May 2018

(C) The Author(s) 2018. This article is published with open access at Springerlink.com DOI: $10.1007 / \mathrm{s} 13320-018-0498-5$

Article type: Regular 
adaptability and low computation cost [9-12]. The CA-CFAR detector achieves the best performance when the background units are IID, and its performance is close to the detection performance of the Neyman-Pearson detector [13]. However, the CA-CFAR is based on the premise that the input samples are IID Gaussian random variables. If the input samples obey Non-IID, the performance of the detector will be significantly reduced.

In this paper, the single-channel data collected by the optical fiber pre-warning system is analyzed. The results show that the background noises collected by the OFPS are normal distribution, but their distribution parameters are not the same, i.e., the distribution of the background noise of the OFPS obeys the Non-IID. Further research shows that the application of the CA-CFAR method to detect harmful intrusion in this case will lead to the decline of detection performance. Therefore, this paper proposes the normalization algorithm to process background noise, which makes the background noise signals obey IID distribution. And then the CA-CFAR method is adopted for the normalized signals to accomplish the harmful intrusions detection. Experiments show that this method can effectively decrease false alarms and improve the detection performance of OFPS.

The rest of this paper is organized as follows. In Section 2, the characteristic analysis of Non-IID for optical signals is introduced. We introduce the detection method of intrusion signals in Section 3. In section 4 the simulation experiment and analysis are presented. Finally, the discussion and conclusion are provided in Section 5.

\section{Characteristic analysis of background noise for the OFPS}

The background noise signals collected by the OFPS obey Non-IID. The detection probability decreases and the false alarm probability increases when the CA-CFAR algorithm is used to detect HI, which affects the detection performance of the system. In order to overcome the problem of the
Non-IID characteristic of the background noise, we need to make use of the mean value and standard deviation from the noise. However, the frequency spectrum characteristics of the HI signal and the background noise signal is different, i.e., the frequency spectrum of the HI signal is in the range of $0 \mathrm{~Hz}-64 \mathrm{~Hz}$, and the background noise signal is distributed at $0 \mathrm{~Hz}-500 \mathrm{~Hz}$. Hence, if the background noise is white noise, the statistical data characteristics such as mean value and standard deviation can be taken from the high frequency band signals of background noise. That will solve the spectrum aliasing between the intrusion signal and background noise. And then the Non-IID characteristic of background noise is converted to IID. This section focuses on the Non-IID analysis and the existence of white noise characteristic about background noise.

\subsection{Non-IID characteristic of background noise}

According to the early studies, it is found that the background noise of OFPS obeys the Gaussian distribution [19]. In order to study the distribution characteristics of background noise further, we collected 40 groups of noise signal, and the temporal resolution of each group is 10 seconds. Then we estimate the variance distribution of each group respectively and plot the frequency distribution histogram, as shown in Fig. 1. The horizontal axis is variance, the left of the vertical axis is the frequency of occurrence, and the right represents the proportion. According to the statistical results, it can be found that there is a large fluctuation in the variance distribution of the background noise signal. What's more, the horizontal axis shows that the difference of variance is up to $30 \%$. As shown in the vertical axis, the probability that the variance is outside the range of $[0.67,0.75]$ is $15 \%$, which means the difference of the distribution characteristic is obvious. Hence, one of the signal characteristics of the OFPS is that the background noise is the Non-IID. 


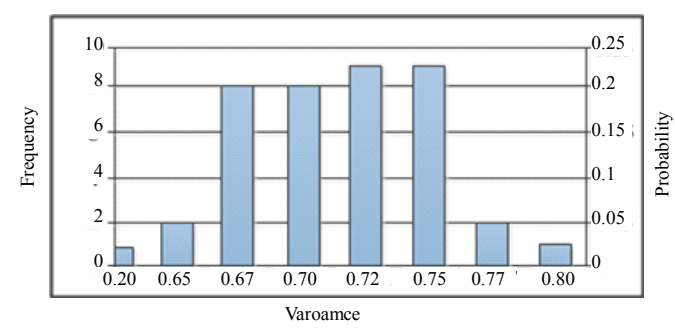

Fig. 1 Statistical result of variance for the noise signals.

\subsection{Analysis of white noise characteristic for the background noise}

This section analyzes the characteristic of background noise in frequency domain by collecting HI signals and pure noise without intrusion, respectively. And the time length of each signal is $10 \mathrm{~s}$. As the sampling frequency of the system is $1 \mathrm{kHz}$, we take 1000 points of the collected signals for fast Fourier transform (FFT), and the spectrum is shown in Fig. 2.

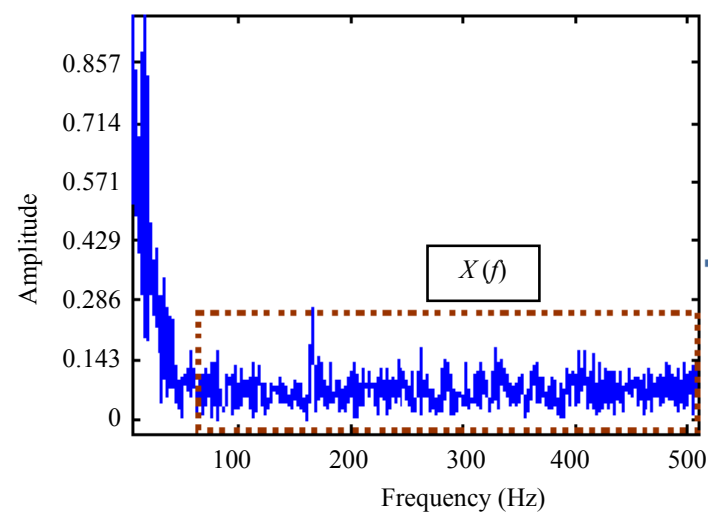

(a)

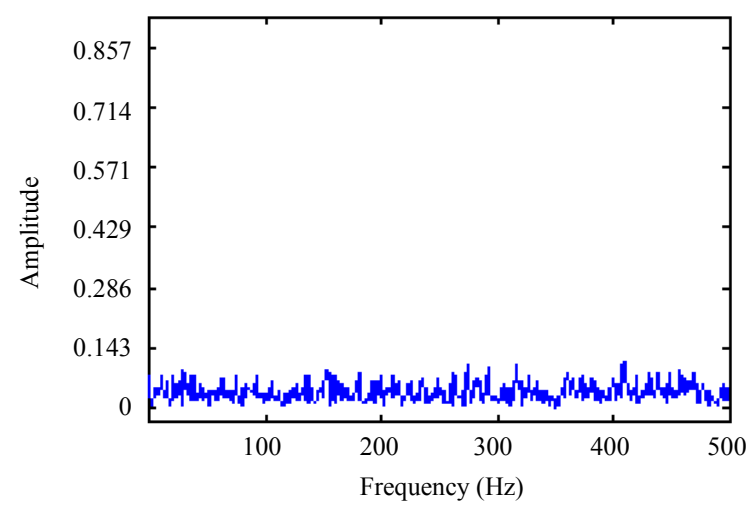

(b)

Fig. 2 Spectra of the acquisition signals: (a) the spectrum of the harmful intrusion and (b) the spectrum of the pure noise.

A spectrogram of signals acquired while walking over an optical fiber is shown in Fig. 2(a). It is found that the amplitude of the HI falls sharply when the frequency is lower than $64 \mathrm{~Hz}$, and it gets relatively stable when the frequency is about $64 \mathrm{~Hz}-500 \mathrm{~Hz}$. We use $X^{\prime}(f)$ to present the frequency part which is higher than $64 \mathrm{~Hz}$. And Fig. 2(b) shows the spectrogram of pure noise without intrusion. From both figures, we can draw a conclusion that the frequency of $\mathrm{HI}$ signals is low, which is concentrated below $64 \mathrm{~Hz}$, and the spectrum of the pure noise signal is relatively homogeneous. In order to obtain the time domain signals represented by $X^{\prime}(f)$, we designed a $64 \mathrm{~Hz}$ high-pass filter for the processing on harmful intrusion. At the same time, the pure noise signal is taken for comparison. Then the mean value and standard deviation are calculated respectively. The signals taken above are all 42 groups, and the statistical results are shown in Fig. 3.

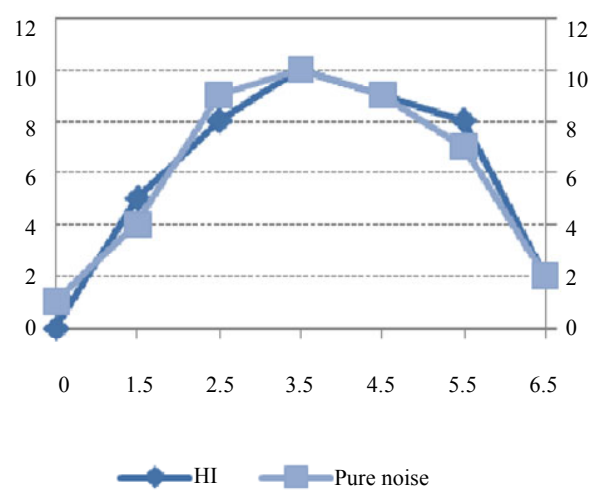

(a)

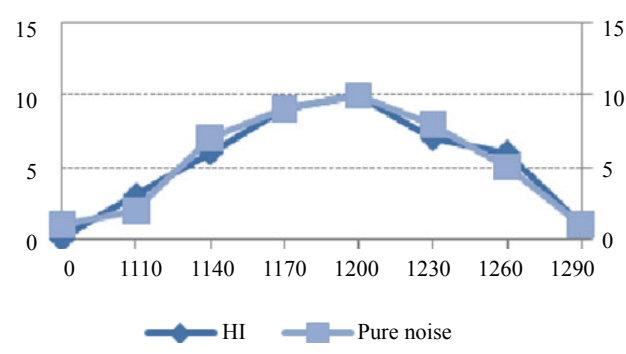

(b)

Fig. 3 Statistical results of two kinds of signal characteristics: (a) means of processed signals and pure noise and (b) standard deviation of processed signals and pure noise.

The statistical results show that the mean value and standard deviation are very close between the pure noise without intrusion and the HI signals with the processing of $64 \mathrm{~Hz}$ high-pass filtering. Because 
they are all subject to Gaussian distribution, it can be reckoned that the distributions of two kinds of signals are the same.

Obviously, based on above analysis, the features of background noise can be summarized as follows: the background noise obeys Non-IID; the background noise of the system is white noise, and we can process the collected $\mathrm{HI}$ with a $64 \mathrm{~Hz}$ high-pass filter, which obtains the signal characteristics to replace the background noise.

\section{Analysis of detection method for the normalized signals}

\subsection{Signal detection flow}

Detection flow of the HI signal for the OFPS is shown in Fig. 4, which mainly includes signal pretreatment, normalization processing, and detection using the CA-CFAR method. Specially, the fiber acquisition signal is binary, so the signal is firstly converted to decimal during the pretreatment process. Secondly, a $64 \mathrm{~Hz}$ low-pass filter is adopted to achieve a higher signal-to-noise ratio (SNR). Then, a $64 \mathrm{~Hz}$ high-pass filter is used to obtain the mean value and variance of the background noise, which is indispensable for the completion of normalization. Finally, we employ the efficient CA-CFAR algorithm for the intrusion detection of above processed signals, thus completing the function of the OFPS.

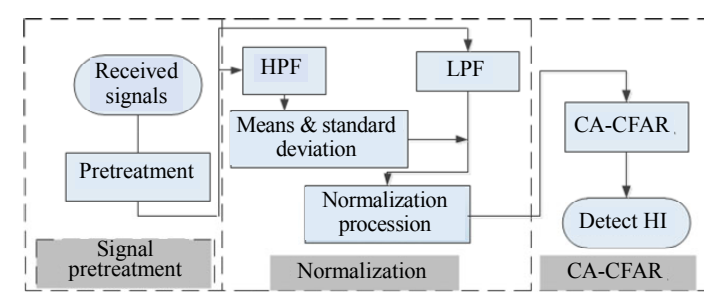

Fig. 4 Principle of detection method.

The distribution conversion for background noise can be achieved through the above process, and the signal detection of harmful intrusion can be completed. Then, the stochastic process analysis method will be used to build the model for the detection process, and the mathematical formula will also be used to analyze the normalization process. Finally, the threshold of the CA-CFAR will be derived.

\subsection{Principle description of intrusion detection}

\subsubsection{Normalization processing for background noise}

The fiber intrusion detection process can be described by a stochastic process model. And the acquisition data of system consists of two parts: the HI signal and the background noise signal, which is expressed as

$$
\left\{\begin{array}{l}
x_{1}(t)=0+n_{1}(t) \\
x_{2}(t)=s_{2}(t)+n_{2}(t) \\
\cdots \\
x_{m}(t)=0+n_{m}(t)
\end{array} \quad m=1,2,3, \cdots\right.
$$

where $s_{2}(t)$ is the harmful intrusion and $n(t)$ is background noise. It is assumed that there exists HI in $s_{2}(t)$, so the values of $s_{1}(t), s_{2}(t), \cdots, s_{m}(t)$ are all 0 . And it is known that background noise $n(t)$ obeys the Non-IID in the analysis of last section, which will affect the detection of HI signal and reduce the detection probability as well as increase the false alarm probability. Hence, normalization is adopted to transform the Non-IID into IID, the expression is as follows:

$$
d(i)=\frac{x(i)-\mu}{\sigma}, \quad i=1,2,3, \cdots
$$

where $x(i)$ is the collected data of the system, $\mu$ is the mean value, $\sigma$ is the standard deviation, and $i$ is time serial number. Theoretically, the mean and standard deviation in this expression should be derived from background noise, which is in the same frequency band of HI signals. However, the above mean and standard deviation cannot be obtained accurately due to the intrusion signal mixed with background noise after the low pass filtered. Meanwhile, it is found that the background noise of the system is white noise through analysis to the acquisition data. Hence, the mean value and 
variance, which are obtained from the collected data with the high-pass filter, are adopted to replace the signal characteristics of the background noise. The result of processing is given as follows:

$$
\left\{\begin{array}{l}
d_{1}(t)=0+n_{1}^{\prime}(t) \\
d_{2}(t)=\frac{s_{2}(t)-\mu}{\sigma}+n_{2}^{\prime}(t), t=1,2,3, \cdots \\
\cdots \\
d_{m}(t)=0+n_{m}^{\prime}(t)
\end{array}\right.
$$

After the normalization, the background noise is transformed into a standard normal distribution with a mean of 0 and standard deviation of 1, i.e., the background noise is a Gaussian random variable that obeys the IID. While HI signal $s_{2}(t)$ becomes $\left(s_{2}(t)-\mu\right) / \sigma$, its amplitude will decrease, the amplitude of the rest position is still 0 . Therefore, it will not affect the HI signal detection due to the difference in amplitude with the changed signals. So the problem of low performance for $\mathrm{HI}$ detection due to the heterogeneous distribution of background noise is solved through the normalization processing.

\subsubsection{Algorithm principle of the CA-CFAR}

Because the acquisition signal after processed obeys IID, the CA-CFAR detection algorithm with high efficiency is used to detect the HI signal. The CFAR algorithm is an adaptive detection method based on the set false alarm probability $P_{f 0}$. The workflow is shown in Fig. 5 .

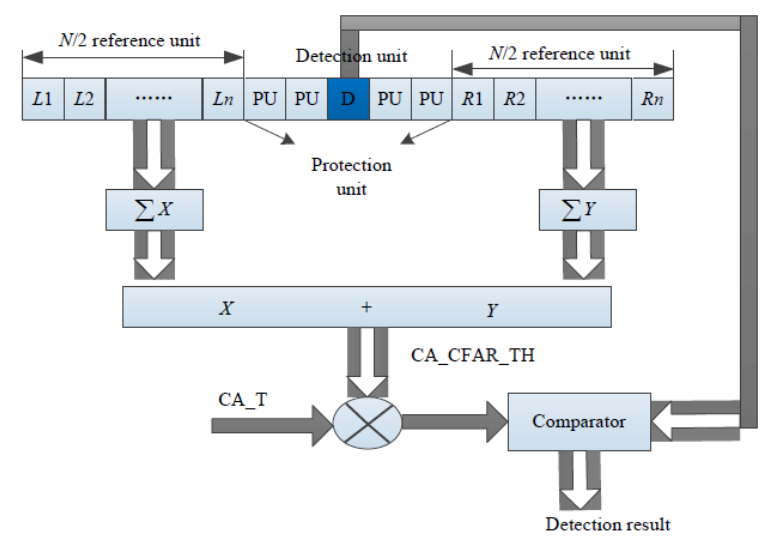

Fig. 5 Block diagram of CA-CFAR detector.
According to our early basic studies to the OFPS, we found that the background is Gaussian noise, and its envelope is Rayleigh distribution. The output signals obey exponential distribution due to the square-law detector. And the probability density function is given as follows:

$$
f(d)=\frac{1}{2 \mu} \exp \left(-\frac{d}{2 \mu}\right), d>0 .
$$

If there is no target, the received signals only contain noise, and $\mu$ is the variance of the noise $\sigma^{2}$. If not, $\mu=\sigma^{2}(1+S)$, where $S$ is the SNR. Moreover, the above signals obey $G\left(N, 2 \sigma^{2}\right)$ based on the IID assumption. Hence, the scale factor of CA-CFAR based on the normalization processing is shown as

$$
T=\left(P_{f 0}\right)^{-1 / 2 N}-1
$$

where $N$ is the number of reference units, $P_{f 0}$ is the false alarm probability. And then the required decision threshold is obtained, so that the detection for HI signal is completed.

In summary, the CA-CFAR algorithm based on the normalization can effectively transform the background noise distribution into IID. That algorithm also can efficiently detect the HI signals and further realize the main function of OFPS.

\section{Experimental analysis}

\subsection{Experimental setup}

The hardware structure and the detection results shown in the system's software for HI signal are given in Fig. 6.

Our group conducted field tests in three regions respectively. During the tests, we collected pure noise signals, shoveling signals, running signals, and picking signals (Case 1 - Case 4). The total length of the optical fiber is 800 meters, and the detailed information is shown in Table 1.

Case 1: The data were collected in the morning in Shangweidian Village. We carried out no operation during the test. Hence, the collected signals were pure noise. 


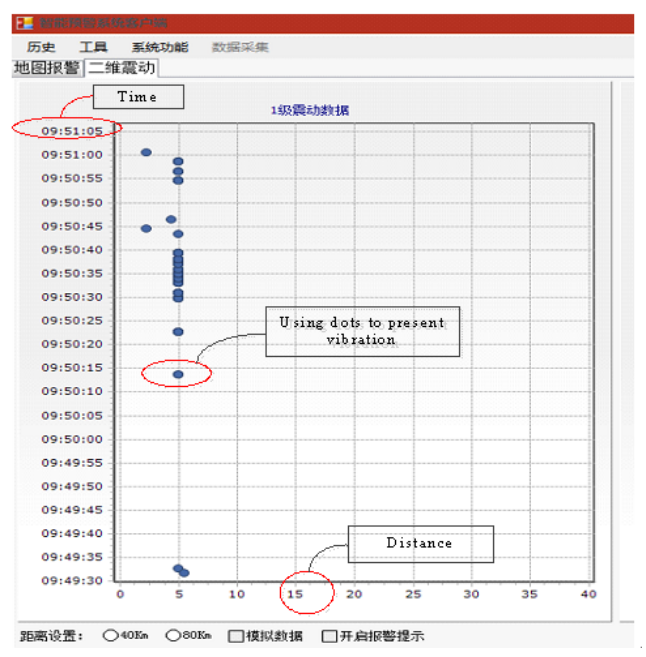

(a)

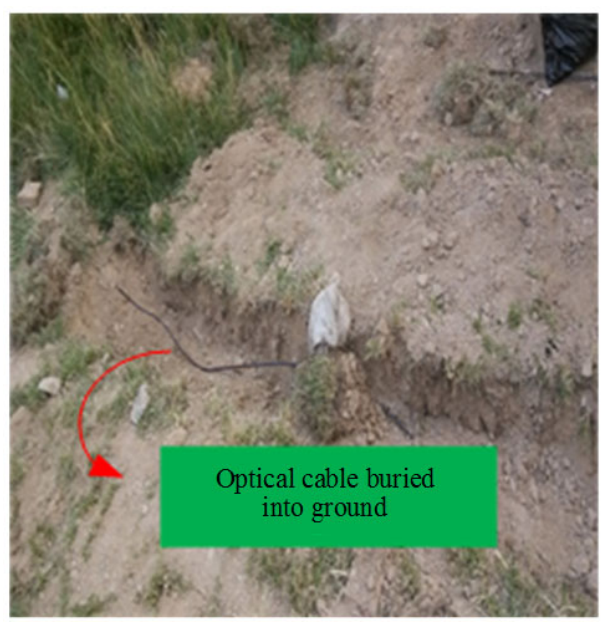

(b)

Fig. 6 Field experiment situation of the system: (a) display interface of the system and (b) buried optical cable at the field.

Table 1 Acquisition signals information.

\begin{tabular}{ccc}
\hline Intrusion signals & $\begin{array}{c}\text { Intrusion location } \\
(\mathrm{m})\end{array}$ & $\begin{array}{c}\text { Intrusion duration } \\
(\mathrm{s})\end{array}$ \\
\hline Case 1 & No & No \\
Case 2 & 500,516 & 5 \\
Case 3 & $466,492,510$ & 5 \\
Case 4 & 492,512 & 5 \\
\hline
\end{tabular}

Case 2: At the $500 \mathrm{~m}$ and $516 \mathrm{~m}$, which were near from the Rushan District, the shovel was used to dig the soil artificially for 8 seconds. Therefore, the collected data was mainly HI signal.

Case 3: We collected data in the afternoon of Baima Village. The tester ran back and forth near the fiber at the $466 \mathrm{~m}, 492 \mathrm{~m}$, and $510 \mathrm{~m}$, and the running continued $8 \mathrm{~s}$.
Case 4: We took the experiment of picking in Rushan District, and the positions of experiment were at $492 \mathrm{~m}$ and $512 \mathrm{~m}$ of the fiber.

\subsection{Non-IID distribution and white noise characteristic analysis of background noise for measured data}

The Non-IID characteristic analysis of the HI signals was taken for the collected signals in Mentougou District, Rushan District, and Guan County, respectively. And we took further analysis on variance, which the statistical results are shown in Table 2.

Table 2 Statistical results of variance in difference areas.

\begin{tabular}{cccc}
\hline $\begin{array}{c}\text { Intrusion } \\
\text { signals }\end{array}$ & Mentougou & Rushan & Guan \\
\hline Case 1 & 0.2416 & 0.2262 & 0.2342 \\
Case 2 & 0.2342 & 0.2235 & 0.2423 \\
Case 3 & 0.2101 & 0.2302 & 0.2000 \\
Case 4 & 0.8000 & 0.2322 & 0.2362 \\
\hline
\end{tabular}

It can be found that the distribution of the signals collected in the field is heterogeneous. Moreover, the variance is intensively located in the range of $[0.2235,0.2362]$, and the frequency of this range is $58 \%$. Hence, the background noise in the field obeys the Non-IID, and this signal characteristic is stable.

The $64 \mathrm{~Hz}$ high-pass filter was adopted for the collected signal in Case 1 - Case 4, and we keept statistics of the variance for the signal, and the results are shown in Table 3.

It can be found from the table that it is similar between the pure noise and HI signal with a $64 \mathrm{~Hz}$ high-pass filter processed. It is indicated that the two distribution parameters are basically same. Meanwhile, the background noise collected by OFPS is white noise.

Table 3 Statistical result of variance.

\begin{tabular}{ccc}
\hline $\begin{array}{c}\text { Type of intrusion } \\
\text { signals }\end{array}$ & Pure noise & $\begin{array}{c}\text { Intrusion signals after } \\
\text { high-pass filtering }\end{array}$ \\
\hline Case 1 & 0.4274 & 0.2000 \\
Case 2 & 0.4084 & 0.8000 \\
Case 3 & 0.4337 & 0.3326 \\
Case 4 & 0.3453 & 0.3768 \\
\hline
\end{tabular}




\subsection{Processing of normalization and detection}

\subsubsection{Analysis of normalization}

The collected signals were processed by the above high-pass filter and then the normalization method was employed to the filtered signals. Hence, the mean value and variance were obtained by the method, which are shown in Table 4. It can be seen from the table that the filtered signals obey the standard normal distribution with the mean of 0 and the variance of 1 . Therefore, the distribution of background noise is converted into IID.

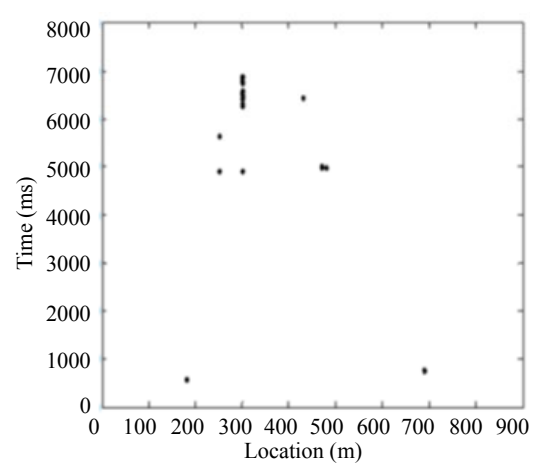

(a1)

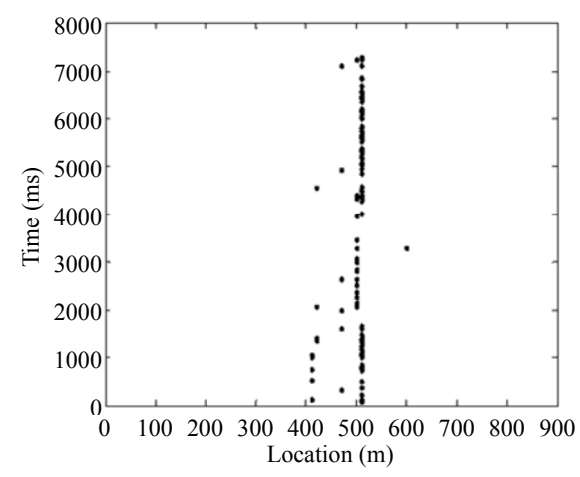

(b1)

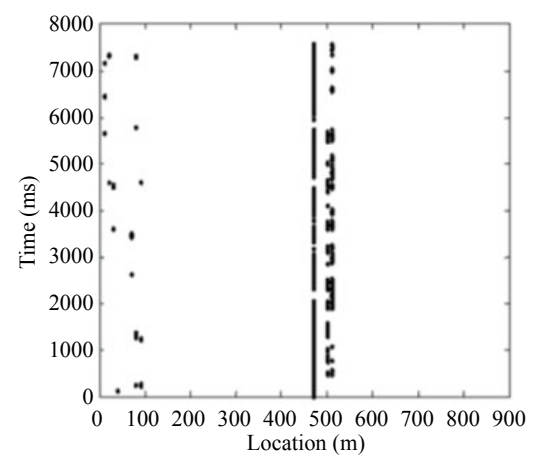

(c1)
Table 4 Statistical result of data characteristic.

\begin{tabular}{ccc}
\hline Measure data & Mean & Standard deviation \\
\hline Case1 & 0.0057 & 0.9427 \\
Case2 & 0.0042 & 0.9917 \\
Case3 & 0.0049 & 0.9420 \\
Case4 & 0.0019 & 0.9783 \\
\hline
\end{tabular}

\subsubsection{Qualitative analysis of test results}

In the above areas, the normalization method was used for the collected signals in Case 1-Case 4. Then, the comparison between the results and the consequence without using the method is taken, and the results are shown Fig. 7.

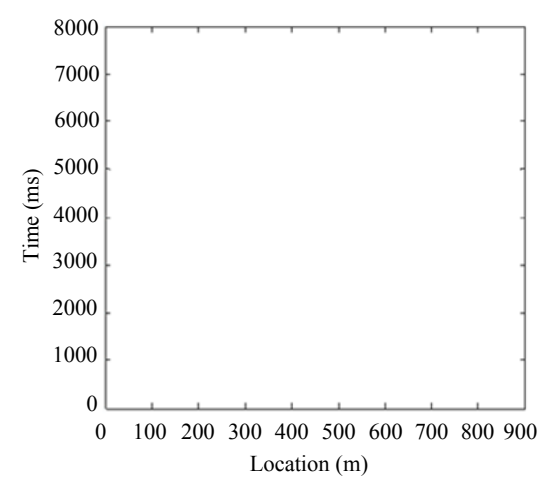

(a2)

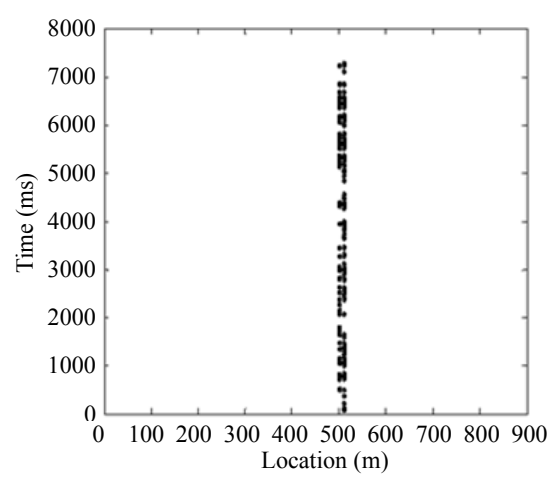

(b2)

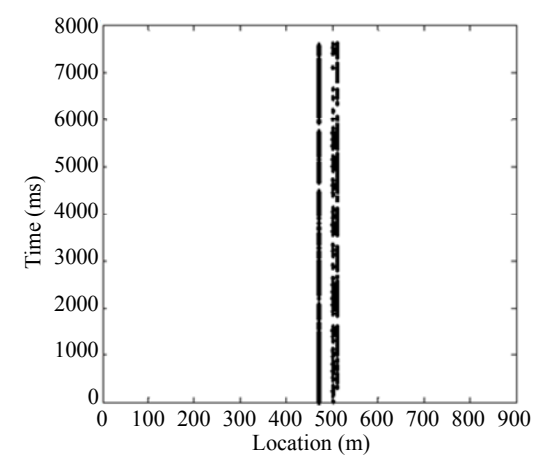

(c2) 


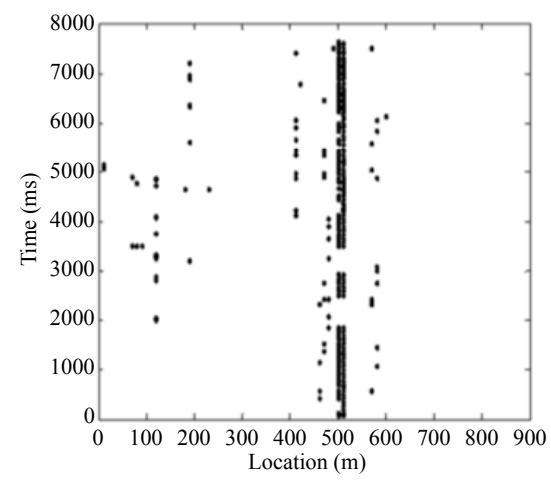

(d1)

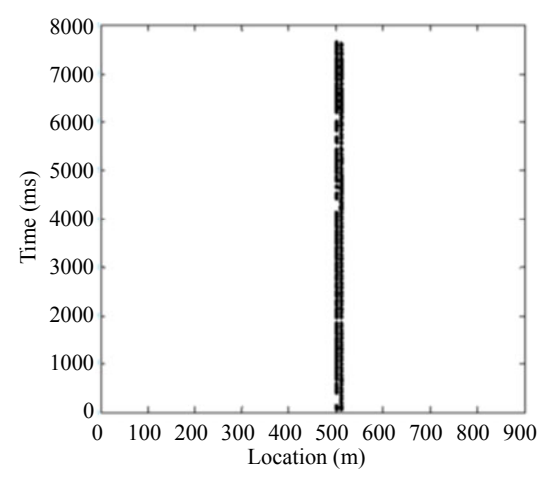

(d2)

Fig. 7 Results of the intrusion signals processed before and after the normalization method: (a) pure noise signals, (b) shoveling signals, (c) running signals, and (d) picking signals.

The following conclusions are obtained according to the above results:

Case 1: After the normalization processing, the false alarm rate is greatly lower than the original signals.

Case 2: The algorithm has a certain detection performance at $516 \mathrm{~m}$, and the algorithm can effectively enhance the detection performance at $500 \mathrm{~m}$ and has a good ability to suppress false alarm at the rest.

Case 3: The algorithm can effectively improve the detection ability of the running position, and the excess alarm point which is not detected by the algorithm is compared with the time domain signal of the corresponding position to prove that it is the false alarm point.

Case 4: The algorithm can obviously improve the vibration detection at $492 \mathrm{~m}$, and it can suppress the performance of false alarm in the rest.

\subsubsection{Quantitative analysis of test results}

We keep counting about the number of correct detection points and the false alarm points, and making comparisons of detection probability and false alarm probability between using the CA-CFAR method based on the normalization processing for background noise and the CA-CFAR method directly adapted to the background noise. Thus the detection gain rate and false alarm rate caused by the algorithm mentioned in this paper can be compared.
Among them, the detection gain rate is calculated by the difference between using the detection probability of the algorithm proposed in this paper and the detection probability that the algorithm is not used. The obtained results are shown in Table 5, where Pfa is the false alarm probability, $\mathrm{Pd}$ is the detection probability, Rdg presents the detection gain rate, and Rfas presents false alarm suppression rate. It can be seen that the probability of detection increases with the use of this algorithm for the signal collected by the OFPS, the false alarm probability is reduced, and the performance of the system is effectively improved.

Table 5 Comparison of detection performance.

\begin{tabular}{ccccc}
\hline $\begin{array}{c}\text { Detection } \\
\text { result }\end{array}$ & Case 1 & Case 2 & Case 3 & Case 4 \\
\hline $\begin{array}{c}P_{\mathrm{fa}} \text { without } \\
\text { normalization }\end{array}$ & $5 \%$ & $4.8 \%$ & $5.2 \%$ & $5.6 \%$ \\
$\begin{array}{c}P_{d} \text { without } \\
\text { normalization }\end{array}$ & $80 \%$ & $82 \%$ & $81 \%$ & $79 \%$ \\
$\begin{array}{c}P_{\mathrm{fa}} \text { with } \\
\text { normalization }\end{array}$ & $1.5 \%$ & $1.0 \%$ & $1.8 \%$ & $1.9 \%$ \\
$\begin{array}{c}P_{d} \text { with } \\
\text { normalization }\end{array}$ & $91 \%$ & $90 \%$ & $92 \%$ & $91 \%$ \\
$R_{\mathrm{dg}}$ & $11 \%$ & $8 \%$ & $11 \%$ & $12 \%$ \\
$R_{\mathrm{fas}}$ & $3.5 \%$ & $3.8 \%$ & $3.4 \%$ & $3.7 \%$ \\
\hline
\end{tabular}

\subsubsection{Analysis of comparative experiment}

In this paper, different CFAR algorithms are used to simulate the HI signal collected by OFPS, and the processing results are shown in Table 6. It can be seen from the table that the detection probability is effectively improved by using the 
CA-CFAR algorithm based on normalization processing compared with other CFAR detection algorithms. The detection probability of the algorithm in this paper is $17 \%$, which is higher than the GO-CFAR algorithm. At the same time, the algorithm can effectively reduce the false alarm probability. Specifically, the false alarm rate is reduced by $3.9 \%$ by using the algorithm proposed in the paper than using the SO-CFAR algorithm, which means the performance of the system is effectively improved. Because the calculation of the average CFAR is small and the time-consuming is low, it is conducive to hardware implementation and meets the real-time processing requirements of the system.

Table 6 Performance comparison of each method.

\begin{tabular}{cccc}
\hline Detection method & $P_{d}$ & $P_{\mathrm{fa}}$ & $\begin{array}{c}\text { Time consuming } \\
(\mathrm{ms})\end{array}$ \\
\hline OS-CFAR & $87.7 \%$ & $3.8 \%$ & 58 \\
GO-CFAR & $74.0 \%$ & $5.3 \%$ & 46 \\
SO-CFAR & $82.2 \%$ & $6.7 \%$ & 39 \\
$\begin{array}{c}\text { CA-CFAR based } \\
\text { on the } \\
\text { normalization }\end{array}$ & $91 \%$ & $2.8 \%$ & 18 \\
\hline
\end{tabular}

\section{Conclusions}

Through the analysis of the vibration signals collected from OFPS experiments, it is found that the background noise presents two characteristics. The background noise obeys the Gaussian distribution, but its distribution of variance is different, so that the distribution of background noise obeys Non-IID. Meanwhile, the noise is presented as white noise characteristics. Therefore, the $64 \mathrm{~Hz}$ high-pass filter is carried out for the HI signals, and the result is alternative with the characteristic of the pure noise signal. Then, the background noise of Non-IID is converted to IID by the normalization algorithm, and the CA-CFAR algorithm is used for the detection of the OFPS and determining whether there are harmful intrusions. The algorithm proposed in this paper is tested for the detection of several common HI signals in three regions. The results show that the false alarm probability is reduced by $3.9 \%$, and the detection probability is increased by $17 \%$.
Open Access This article is distributed under the terms of the Creative Commons Attribution 4.0 International License (http://creativecommons.org/licenses/by/4.0/), which permits unrestricted use, distribution, and reproduction in any medium, provided you give appropriate credit to the original author(s) and the source, provide a link to the Creative Commons license, and indicate if changes were made.

\section{References}

[1] W. Liang, L. L. Lu, and L. B. Zhang, "Coupling relations and early-warning for 'equipment chain' in long-distance pipeline," Mechanical Systems and Signal Processing, 2013, 41(1-2): 335-347.

[2] J. W. Qiu, W. Liang, L. B. Zhang, X. H. Yu, and M. Zhang, "The early-warning model of equipment chain in gas pipeline based on DNN-HNM," Journal of Natural Gas Science \& Engineering, 2015, 27: 1710-1722.

[3] K. Jing and Z. H. Zou, "Time prediction model for pipeline leakage based on grey relational analysis," Physics Procedia, 2012, 25(2): 2019-2024.

[4] Y. X. Zhang, Y. M. Xu, Y. Y. Shan, Z. H. Sun, F. Zhu, and X. P. Zhang, "Polarization dependence of phase-sensitive optical time-domain reflectometry and its suppression method based on orthogonal-state," Optical Engineering, 2016, 55(7): 074109-1- 074109-6.

[5] S. Liang, X. Z. Sheng, S. Q. Lou, Y. Feng, and K. N. Zhang, "Combination of phase-sensitive OTDR and Michchelson interferometer for nuisance alarm rate reduction," IEEE Photonics Journal, 2016, 8(2): $1-12$.

[6] H. J. Wu, S. K. Xiao, X. Y. Li, Z. N. Wang, J. W. Xu, and Y. J. Rao, "Separation and determination of the disturbing signals in phase-sensitive optical time domain reflectometry ( $\Phi-O T D R)$," Journal of Lightwave Technology, 2015, 33(15): 3156-3162.

[7] S. R. Xie and M. Zhang, "Ultra long distance distributed fiber-optic system for intrusion detection," Advanced Sensor Systems \& Applications $\quad V, \quad 2012, \quad 8561(3): \quad 85611 \mathrm{~W}-1-$ 85611W-6.

[8] H. M. Finn and R. S. Johnson, "Adaptive detection mode with threshold control as a function of spatially sampled clutter-level estimates," $R C A$ Review, 1968.

[10] F. K. Bi, C. Feng, H. Q. Qu, and T. Zheng, "Harmful intrusion detection algorithm of optical fiber pre-warning system based on correlation of orthogonal polarization signals," Photonic Sensors, 2017, 7(3): 226-233. 
[10] Z. Qiu, T. Zheng, H. Q. Qu, and L. P. Pang, "A new method based on CFAR and DE for OFPS," Photonic Sensors, 2016, 6(3): 261-267.

[11] F. K. Bi, X. C. Ren, H. Q. Qu, and R. Q. Jiang, “A two-level detection algorithm for optical fiber vibration," Photonic Sensors, 2015, 5(3): 284-288.

[12] H. Qu, X. Ren, G. Li, Y. Li, and C. Zhang, "Study on the algorithm of vibration source identification based on the optical fiber vibration pre-warning system,"
Photonic Sensors, 2015, 5(2): 180-188.

[13] A. Zaimbashi, "An adaptive cell averaging-based CFAR detector for interfering targets and clutter-edge situations," Digital Signal Processing, 2014, 31(5): 59-68.

[14] H. Q. Qu, T. Zheng, L. P. Pang, and X. L. Li, "A new two-dimensional method to detect harmful intrusion vibrations for optical fiber pre-warning system," Optik, 2016, 127(10): 4461-4469. 